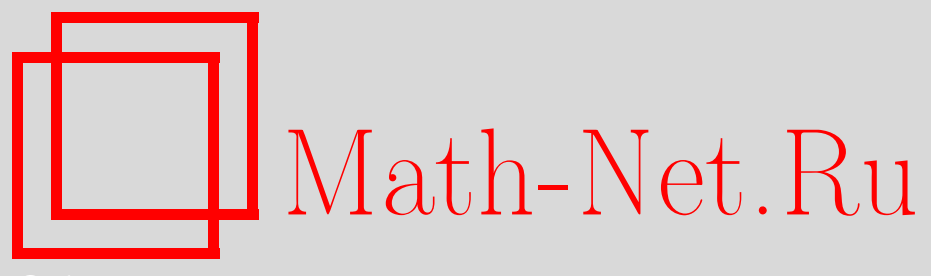

А. А. Ершов, Асимтотическое разложение решения дифференциального уравнения второго порядка, Матем. заметки, 2009, том 85, выпуск 1, 134-138

DOI: https://doi.org/10.4213/mzm6587

Использование Общероссийского математического портала Math-Net.Ru подразумевает, что вы прочитали и согласны с пользовательским соглашением http://www . mathnet.ru/rus/agreement

Параметры загрузки:

IP: 18.209 .158 .208

26 апреля 2023 г., 10:17:36

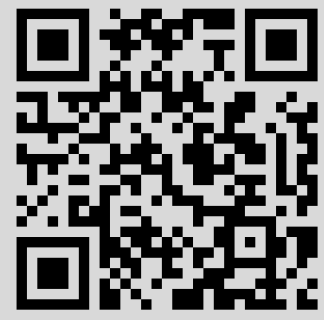




\section{Асимтотическое разложение решения дифференциального уравнения второго порядка}

\section{А. А. Ершов}

Введение. Рассматривается дифференциальное уравнение второго порядка

$$
\frac{d^{2} u}{d t^{2}}+Q(t) u=0
$$

Известно, что при достаточно широких предположениях относительно комплекснозначной функции вещественной переменной $Q(t)$ можно получить асимптотические разложения решений этого уравнения при больших значениях аргумента $t$. Так называемый метод ВКБ позволяет получать такие разложения, например, в случае, когда модули коэффициента $Q(t)$ и его производных любого порядка стремятся к бесконечности при $t \rightarrow \infty[1]$.

1. В этом пункте рассматривается случай, когда

$$
Q(t)=P_{n}(t)+\varphi(t),
$$

где $P_{n}(t)$ - алгебраический многочлен степени $n$, а $\varphi(t)$ - почти периодическая функция вида $\sum_{k=1}^{\infty} c_{k} e^{i \lambda_{k} t}, c_{k}, \lambda_{k} \in \mathbb{R}$. Задача состоит в том, чтобы доказать существование асимптотического разложения решения при большом аргументе вида

$$
u(t) \stackrel{\text { as }}{=} Q(t)^{-1 / 4} \exp (i S(t))\left(1+\sum_{j=1}^{\infty} \frac{w_{j}(t)}{t^{j}}\right),
$$

где $S(t)=\int_{0}^{t} \sqrt{Q(s)} d s$, а $w_{j}(t)$ - некоторые почти периодические функции вида $\sum_{j=1}^{\infty} b_{j, k} \times$ $e^{i \lambda_{j, k} t}$.

Для дальнейших поисков решения введем класс функций $V$, замкнутый относительно дифференцирования, умножения и взятия линейных комбинаций. Потребуем также, чтобы интеграл с переменным верхним пределом, среднее значение подынтегральной функции которого равно нулю, принадлежал $V$.

Примером такого класса могут быть функции вида

$$
f(t)=\sum_{k=1}^{\infty} c_{k} e^{i \lambda_{k} t},
$$

где $\lambda_{k}>\varepsilon>0$ для любого натурального $k$ и $\sum_{k=1}^{\infty}\left|c_{k} \lambda_{k}^{n}\right|<\infty$ для любого целого $n$. Например, функция $f(t)=\left(3-e^{i t}-e^{i \sqrt{2} t}\right)^{-1}$ принадлежит этому классу. Легко доказать, что множество таких функций действительно замкнуто относительно вышеуказанных операций.

Будем считать, что функция $\varphi$ принадлежит классу $V$.

Будем исследовать формальное асимптотическое разложение решения, сделав замену неизвестной функции:

$$
u(t)=w(t) Q(t)^{-1 / 4} \exp (i S(t)) .
$$

Функция $w(t)$ удовлетворяет уравнению

$$
\frac{d^{2} w}{d t^{2}}+\frac{d w}{d t}\left(2 i Q(t)^{1 / 2}-\frac{1}{2} \frac{Q^{\prime}(t)}{Q(t)}\right)+w\left(\frac{5}{16} \frac{\left[Q^{\prime}(t)\right]^{2}}{[Q(t)]^{2}}-\frac{1}{4} \frac{Q^{\prime \prime}(t)}{Q(t)}\right)=0 .
$$

Работа выполнена при поддержке Российского фонда фундаментальных исследований (грант № 07-01-96002 р-урал-а).

(C) A. A. Ершов, 2009 
Рассмотрим полином четной степени $P_{2 m}(t)$, и пусть его старший коэффициент равен 1 (это не нарушает общности рассуждений, исследование в случае полинома нечетной степени по существу не отличается от рассмотренного ниже). Пусть $P_{2 m}(t)=\sum_{k=0}^{2 m} a_{k} t^{k}$, где $a_{2 m}=1, m$ - натуральное число. Тогда можно получить следующие разложения в ряд коэффициентов уравнения (2). Например,

$$
\begin{aligned}
Q(t)^{1 / 2} & =\sqrt{t^{2 m}+\sum_{k=0}^{2 m-1} a_{k} t^{k}+\varphi(t)} \\
& =t^{m}\left(1+\frac{1}{2}\left(\sum_{k=0}^{2 m-1} a_{k} t^{k-2 m}+t^{-2 m} \varphi(t)\right)-\frac{1}{8}\left(\sum_{k=0}^{2 m-1} a_{k} t^{k-2 m}+t^{-2 m} \varphi(t)\right)^{2}+\cdots\right) .
\end{aligned}
$$

Так как класс $V$ замкнут относительно умножения и взятия линейных комбинаций, уравнение (2) можно переписать следующим образом:

$$
\frac{d^{2} w}{d t^{2}}+\frac{d w}{d t}\left(2 i t^{m}+\sum_{k=-m+1}^{\infty} \frac{1}{t^{k}} \varphi_{k}(t)\right)+w\left(\sum_{k=2}^{\infty} \frac{1}{t^{k}} \psi_{k}(t)\right)=0
$$

где $\varphi_{k}(t), \psi_{k}(t)$ являются функциями из класса $V$, а ряды сходятся. Первые члены рядов $\varphi_{-m+1}, \ldots, \varphi_{m-1}$ и $\psi_{2}, \ldots, \psi_{2 m-1}$ даже постоянные.

Будем искать решение в виде формального ряда

$$
w=1+\frac{w_{m+1}}{t^{m+1}}+\frac{w_{m+2}}{t^{m+2}}+\frac{w_{m+3}}{t^{m+3}}+\frac{w_{m+4}}{t^{m+4}}+\cdots,
$$

где $w_{i}$ - ограниченные функции.

Хотя все функции $w_{k}$ при $k<3 m$ оказываются постоянными, для однообразности доказательства обобщим все $w_{i}$ до почти периодических функций класса $V$.

После формального дифференцирования этого ряда получаем соотношения

$$
\begin{aligned}
w^{\prime} & =\frac{w_{m+1}^{\prime}}{t^{m+1}}-(m+1) \frac{w_{m+1}}{t^{m+2}}+\frac{w_{m+2}^{\prime}}{t^{m+2}}-(m+2) \frac{w_{m+2}}{t^{m+3}}+\frac{w_{m+3}^{\prime}}{t^{m+3}}-(m+3) \frac{w_{m+3}}{t^{m+4}}+\cdots, \\
w^{\prime \prime} & =\frac{w_{m+1}^{\prime \prime}}{t^{m+1}}-2(m+1) \frac{w_{m+1}^{\prime}}{t^{m+2}}+(m+1)(m+2) \frac{w_{m+1}}{t^{m+3}}+\frac{w_{m+2}^{\prime \prime}}{t^{m+2}}-2(m+2) \frac{w_{m+2}^{\prime}}{t^{m+3}}+\cdots .
\end{aligned}
$$

Приравняем функции при равных степенях $t$ :

$$
\begin{gathered}
t^{-1}: 2 i w_{m+1}^{\prime}=0, \quad w_{m+1}=\alpha_{m+1}, \\
t^{-2}: \quad 2 i\left(-(m+1) w_{m+1}+w_{m+2}^{\prime}\right)+w_{m+1}^{\prime} \varphi_{1}(t)+\psi_{2}(t)=0, \\
-2 i(m+1) \alpha_{m+1}+2 i w_{m+2}^{\prime}+\psi_{2}(t)=0, \\
w_{m+2}^{\prime}=(m+1) \alpha_{m+1}-\frac{1}{2 i} \psi_{2}(t)=\frac{i}{2} \psi_{2}(t)+(m+1) \alpha_{m+1}=\chi_{m+2}(t)+(m+1) \alpha_{m+1}, \\
w_{m+2}=\int_{0}^{t} \chi_{m+2}(\tau) d \tau+(m+1) \alpha_{m+1} t+\alpha_{m+2} .
\end{gathered}
$$

Найдем $\alpha_{m+1}$ из условия

$$
(m+1) \alpha_{m+1} t+\int_{0}^{t} \frac{i}{2} \psi_{2}(\theta) d \theta=O(1), \quad t \rightarrow \infty .
$$

Из выбора $\alpha_{m+1}$ следует, что среднее значение функции $\chi_{m+2}(t)$ равно нулю, поэтому $w_{m+2}(t)$ будет принадлежать классу $V$.

Константа $\alpha_{m+2}$ пока еще не определена, мы определим ее из следующих разложений:

$$
\begin{gathered}
t^{-3}: \quad 2 i\left(-(m+2) w_{m+2}+w_{m+3}^{\prime}\right)+w_{m+1}^{\prime} \varphi_{2}(t)+w_{m+2}^{\prime} \varphi_{1}(t)+\psi_{3}(t)=0, \\
w_{m+3}^{\prime}=(m+2) w_{m+2}+\frac{i}{2}\left(w_{m+1}^{\prime} \varphi_{2}+w_{m+2}^{\prime} \varphi_{1}+\psi_{3}\right)=\chi_{m+3}(t)+(m+2) \alpha_{m+2}
\end{gathered}
$$

(обозначим за $\chi_{m+3}$ соответствующую функцию). 
Аналогично

$$
w_{m+3}=\int_{0}^{t} \chi_{m+3}(\tau) d \tau+(m+2) \alpha_{m+2} t+\alpha_{m+3} .
$$

Функция $\chi_{m+3}$ состоит из произведений $\varphi, \varphi^{\prime}, \varphi^{\prime \prime}$, первообразных этих функций и их линейных комбинаций, но поскольку функция $\varphi=\sum_{k=1}^{\infty}\left(p_{k} \sin \widetilde{\lambda}_{k} t+q_{k} \cos \widetilde{\lambda}_{k} t\right)$, то $\chi_{m+3}$ такого же вида и, следовательно, из класса $V$. Значит, константу $(m+2) \alpha_{m+2}$ можно также найти как среднее значение функции $\left(-\chi_{m+3}\right)$, т.е.

$$
\alpha_{m+2}=-\frac{1}{m+2} \lim _{T \rightarrow \infty} \frac{1}{T} \int_{0}^{T} \chi_{m+3}(\tau) d \tau .
$$

Заметим, что для почти периодических функций этот предел равен среднему значению функции $-(1 /(m+2)) \chi_{m+3}$. Функция $w_{m+3}$ будет также из класса $V$.

Рассмотрим произвольную степень $-p$, где $p$ - натуральное число:

$$
\begin{gathered}
t^{-p}: w_{p}^{\prime \prime}-2(p-1) w_{p-1}^{\prime}+(p-2)(p-1) w_{p-2}+2 i\left(w_{m+p}^{\prime}-(m+p-1) w_{m+p-1}\right) \\
\quad+\sum_{j=m+1}^{p+m-1}\left(w_{j}^{\prime}-(j-1) w_{j-1}\right) \varphi_{p-j}+\sum_{k=2}^{p-m-1} \psi_{k} w_{p-k}+\psi_{p}=0, \\
w_{m+p}^{\prime}=(m+p-1) w_{m+p-1} \\
\quad+\frac{i}{2}\left(w_{p}^{\prime \prime}-2(p-1) w_{p-1}^{\prime}+(p-2)(p-1) w_{p-2}\right. \\
\left.\quad+\sum_{j=m+1}^{p+m-1}\left(w_{j}^{\prime}-(j-1) w_{j-1}\right) \varphi_{p-j}+\sum_{k=2}^{p-m-1} \psi_{k} w_{p-k}+\psi_{p}\right) \\
=\chi_{m+p}(t)+(m+p-1) \alpha_{m+p-1}, \\
w_{m+p}(t)=\int_{0}^{t} \chi_{m+p}(\tau) d \tau+(m+p-1) \alpha_{m+p-1} t+\alpha_{m+p},
\end{gathered}
$$

где

$$
\alpha_{m+p-1}=-\frac{1}{(m+p-1)} \lim _{T \rightarrow \infty} \frac{1}{T} \int_{0}^{T} \chi_{m+p}(\tau) d \tau .
$$

Эта формула будет верна для любой функции $w_{i}$. При этом надо полагать все $w_{i} \equiv 0$, если $i<m+1$.

Таким образом, следуя данному алгоритму, найдем бесконечный асимптотический ряд, являющийся формальным. Можно доказать, что существует решение уравнения, для которого данный ряд является асимптотическим.

2. Заметим, что можно рассмотреть и другие случаи быстро растущего $Q(t)$. Например, когда $Q(t)=\exp (2 \lambda t)+\varphi(t)$, где $\lambda \in \mathbb{R}, \lambda>0$, а $\varphi(t)$ - некоторая гладкая функция, имеющая более медленный рост. Но в этом случае уже будет отсутствовать резонанс. Докажем существование асимптотического разложения решения при большом аргументе вида

$$
u(t) \stackrel{\text { as }}{=} Q(t)^{-1 / 4} \exp (i S(t))\left(1+\sum_{j=1}^{\infty} \frac{w_{j}(t)}{e^{j \lambda t}}\right),
$$

где $S(t)=\int_{0}^{t} \sqrt{Q(s)} d s$, а $w_{j}(t)$ - некоторые функции, растущие медленнее экспоненты $c_{j} e^{j \mu t}, 0<\mu<\lambda$.

С этой целью рассмотрим следующее семейство классов функций $W_{k}$. Класс $W_{k}$ зададим условием: гладкая функция $f(t)$ принадлежит классу $W_{k}$, если для любого целого неотрицательного $n$ существует константа $C_{n}$, зависящая от $k$, такая, что $\left|f^{(n)}(t)\right|<C_{n} e^{k \mu t}$ для любого $t>0$. Из определения класса $W_{k}$ следует, что интеграл с переменным верхним пределом от функции из $W_{k}$ принадлежит $W_{k}$. Также заметим, 
что если функции $f$ и $g$ принадлежат классам $W_{i}$ и $W_{j}$ соответственно, то произведение $f * g$ будет принадлежать классу $W_{i+j}$.

Такими функциями могут быть алгебраические или тригонометрические многочлены.

В дальнейшем будем считать, что функция $\varphi$ принадлежит классу $W_{1}$.

Чтобы найти формальное асимптотическое разложение решения, выполним ту же замену функции:

$$
u(t)=w(t) Q(t)^{-1 / 4} \exp (i S(t)) .
$$

Функция $w(t)$ удовлетворяет уравнению (2)

Рассмотрим экспоненту с произвольным показателем, представив ее в виде $e^{2 \lambda t}$, где $\lambda$ любое положительное число. Получим разложения в ряд коэффициентов уравнения (2). Например,

$$
\begin{aligned}
Q(t)^{1 / 2} & =e^{\lambda t} \sqrt{1+\frac{\varphi(t)}{e^{2 \lambda t}}}=e^{\lambda t}\left(1+\frac{1}{2} \frac{\varphi(t)}{e^{2 \lambda t}}-\frac{1}{8} \frac{\varphi(t)^{2}}{e^{4 \lambda t}}+\cdots\right), \\
\frac{1}{Q(t)} & =\frac{1}{e^{2 \lambda t}} \frac{1}{1+\varphi(t) / e^{2 \lambda t}}=\frac{1}{e^{2 \lambda t}}\left(1-\frac{\varphi(t)}{e^{2 \lambda t}}+\frac{(\varphi(t))^{2}}{e^{4 \lambda t}}-\cdots\right) .
\end{aligned}
$$

Учитывая то, что все классы $W_{k}$ замкнуты относительно взятия линейных комбинаций, и свойство произведения функций из разных классов, уравнение (2) преобразуем к следующему виду:

$$
\frac{d^{2} w}{d t^{2}}+\frac{d w}{d t}\left(2 i e^{\lambda} t+\sum_{k=0}^{\infty} \frac{1}{e^{k \lambda t}} \varphi_{k}(t)\right)+w\left(\sum_{k=0}^{\infty} \frac{1}{e^{k \lambda t}} \psi_{k}(t)\right)=0
$$

где $\varphi_{0}(t), \psi_{0}(t) \in W_{1}, \varphi_{k}(t), \psi_{k}(t) \in W_{k}$ при $k>0$, а ряды являются сходящимися.

Решение найдем в виде формального ряда

$$
w^{\prime}=\frac{w_{1}}{e^{\lambda t}}+\frac{w_{2}}{e^{2 \lambda t}}+\frac{w_{3}}{e^{3 \lambda t}}+\frac{w_{4}}{e^{4 \lambda t}}+\cdots,
$$

где $w_{i}$ - функции, принадлежащие классам $W_{i}$.

Выполнив формальное дифференцирование этого ряда, получим соотношения

$$
\begin{aligned}
w^{\prime} & =\frac{w_{1}^{\prime}-\lambda w_{1}}{e^{\lambda t}}+\frac{w_{2}^{\prime}-2 \lambda w_{2}}{e^{2 \lambda t}}+\frac{w_{3}^{\prime}-3 \lambda w_{3}}{e^{3 \lambda t}}+\cdots, \\
w^{\prime \prime} & =\frac{w_{1}^{\prime \prime}-2 \lambda w_{1}^{\prime}+\lambda^{2} w_{1}}{e^{\lambda t}}+\frac{w_{2}^{\prime \prime}-4 \lambda w_{1}^{\prime}+4 \lambda^{2} w_{1}}{e^{2 \lambda t}}+\cdots .
\end{aligned}
$$

Приравняем функции при одинаковых показателях экспоненты:

$$
\begin{gathered}
e^{0}: \quad 2 i\left(w_{1}^{\prime}-\lambda w_{1}\right)+\psi_{0}(t)=0, \\
w_{1}^{\prime}=\lambda w_{1}+\frac{i}{2} \psi_{0}(t), \quad w_{1}^{\prime}=\lambda w_{1}, \quad w_{1 \text { odn. }}=c e^{\lambda t}, \quad w_{1}=c(t) e^{\lambda t}, \\
c^{\prime}(t) e^{\lambda t}=\frac{i}{2} \psi_{0}(t), \quad c(t)=\frac{i}{2} \int_{\alpha}^{t} \psi_{0} e^{-\lambda t} d \tau+C \\
w_{1}(t)=\left(\frac{i}{2} \int_{\alpha}^{t} \psi_{0} e^{-\lambda \tau} d \tau+C\right) e^{\lambda t} .
\end{gathered}
$$

Для того чтобы ряд имел смысл, функция $w_{1}$ должна расти медленнее знаменателя или, что практически то же самое, принадлежать классу $W_{1}$. Если функция $\psi_{0}$ больше некоторой положительной константы, начиная с некоторого $t$, но принадлежит классу $W_{1}$, то единственным способом сохранения корректности ряда является положить $C=0$ и $\alpha=+\infty$. В этом случае функция $w_{1}$ будет принадлежать классу $W_{1}$. Получаем

$$
\begin{gathered}
e^{-\lambda t}: \quad w_{1}^{\prime \prime}-2 \lambda w_{1}^{\prime}+\lambda^{2} w_{1}+2 i\left(w_{2}^{\prime}-2 \lambda w_{2}\right)+\left(w_{1}^{\prime}-\lambda w_{1}\right) \varphi_{0}+\psi_{1}+w_{1} \psi_{0}=0, \\
w_{2}^{\prime}=2 \lambda w_{2}+\frac{i}{2}\left(w_{1}^{\prime \prime}-2 \lambda w_{1}^{\prime}+\lambda^{2} w_{1}+\left(w_{1}^{\prime}-\lambda w_{1}\right) \varphi_{0}+\psi_{1}+w_{1} \psi_{0}\right)=2 \lambda w_{2}+\chi_{2}(t),
\end{gathered}
$$


где

$$
\begin{aligned}
& \chi_{2}(t)=\frac{i}{2}\left(w_{1}^{\prime \prime}-2 \lambda w_{1}^{\prime}+\lambda^{2} w_{1}+\left(w_{1}^{\prime}-\lambda w_{1}\right) \varphi_{0}+\psi_{1}+w_{1} \psi_{0}\right), \quad w_{2}=c(t) e^{2 \lambda t}, \\
& c(t)=\int_{+\infty}^{t} \chi_{2} e^{-2 \lambda \tau} d \tau+C, \quad w_{2}(t)=\left(\frac{i}{2} \int_{+\infty}^{t} \chi_{2} e^{-2 \lambda \tau} d \tau+C\right) e^{2 \lambda t}, \quad C=0 .
\end{aligned}
$$

Все функции, составляющие $\chi_{2}$, принадлежат классу $W_{1}$. Легко показать, что любая производная $\chi_{2}$ растет медленнее экспоненты $e^{2 \mu t}$, а значит, $\chi_{2}$ и $w_{2}$ принадлежат классу $W_{2}$.

Рассмотрим произвольный показатель $-k \lambda$, где $k$ - натуральное число:

$$
\begin{gathered}
e^{-k \lambda t}: w_{k}^{\prime \prime}-2 k \lambda w_{k}^{\prime}+k^{2} \lambda^{2} w_{k}+2 i\left(w_{k+1}^{\prime}-k \lambda w_{k}\right) \\
+\sum_{j=1}^{k}\left(w_{j}^{\prime}-j \lambda w_{j}\right) \varphi_{k-j}+\psi_{k}+\sum_{j=1}^{k} w_{j} \psi_{k-j}=0, \\
w_{k+1}^{\prime}=k \lambda w_{k}+\frac{i}{2}\left(w_{k}^{\prime \prime}-2 k \lambda w_{k}^{\prime}+k^{2} \lambda^{2} w_{k}+\sum_{j=1}^{k}\left(w_{j}^{\prime}-j \lambda w_{j}\right) \varphi_{k-j}+\psi_{k}+\sum_{j=1}^{k} w_{j} \psi_{k-j}\right) \\
=k \lambda w_{k}+\chi_{m+1}(t), \\
w_{k+1}=c(t) e^{k \lambda t}, \quad c(t)=\int_{+\infty}^{t} \chi_{k+1} e^{-k \lambda \tau} d \tau+C, \\
w_{k+1}(t)=\frac{i}{2} e^{k \lambda t} \int_{+\infty}^{t} \chi_{k+1} e^{-k \lambda \tau} d \tau, \quad C=0 .
\end{gathered}
$$

Следовательно, в данном случае также построен бесконечный асимптотический ряд. Также можно доказать, что существует решение, разлагающееся в этот ряд.

Автор выражает глубокую благодарность А. М. Ильину за постановку задачи и внимание к работе.

\section{СПИСОК ЦИТИРОВАННОЙ ЛИТЕРАТУРЫ}

[1] М.В. Федорюк, Асимптотические методы для линейных обыкновенных дифференциальных уравнений, Справочная математическая библиотека, Наука, М., 1983.

\section{А. А. Ершов}

Челябинский государственный университет

E-mail: ale10919@yandex.ru
Поступило 06.06.2008 